P\&A Año 2, N. ${ }^{\circ} 3$

julio-diciembre 2017

pp. [5]-15

\title{
Resumen
}

La necesidad de plantear una nueva estrategia de aprendizaje que permita a los estudiantes un mayor compromiso con las actividades que se desarrollan en la asignatura de Diseño Urbano I de manera que puedan adquirir nuevos conocimientos con profundidad y permanencia; amerita el planteamiento de una estrategia que promueva el incremento de la construcción del conocimiento interno y activo del individuo, que se inicia en el contexto social de cada estudiante. La construcción del nuevo conocimiento tiene en cuenta los conocimientos previos en el desarrollo de una secuencia instruccional.

En esta asignatura se toma el caso "Mi barrio" para incorporar lo exógeno, la realidad compleja en el desarrollo instruccional. El estudiante asume el empoderamiento de su aprendizaje cuando presenta su barrio en una secuencia de contenidos para lograr las competencias, en la que el docente, integrante de una construcción conjunta en el proceso de aprendizaje; se convierte en el agente motivador para incrementar el nivel de logro. Para la evaluación del aprendizaje se aplica la taxonomía "SOLO" de Biggs en los niveles Multiestructural, relacional y abstracto ampliado.

Palabras claves: Aprendizaje, competencia, estrategia contexto social, taxonomía "SOLO”.

\section{Docencia en arquitectura: Constructivismo y estrategia de contexto social para logro de competencias*}

\author{
Teaching Architecture: Constructivism strategy social Context to achieve \\ competence
}

\author{
Arq. Soledad Guadalupe Herrera García**
}

Recibido: 16 de abril de 2017

Aceptado: 29 de junio de 2017

\begin{abstract}
The necessity to establish a new learning strategy that allows students to become more involved in activities developed in the subject of Urban Design I, in order to acquire new knowledge, with depth and permanence, merits to propose a strategy that promotes the increase in the construction of internal and active knowledge; which begins in the social context of each student. The Construction of new knowledge requires the previous knowledge in developing an instructional sequence.

In this subject it is considered the "Mi barrio" case, in order to incorporate the exogenous, the complex reality in instructional development. The student assumes the empowerment of his learning, when he shows his neighborhood in a sequence of contents to achieve the competence, where the teacher, who is part of a joint construction in the learning process; becomes in the motivating agent to increase the level of achievement. For assessment of learning, it is applied "SOLO" taxonomy from Biggs at the multi structural, relational and extended abstract levels.
\end{abstract}

Keywords: Learning, competition, strategy, SOLO taxonomy

\footnotetext{
* El artículo recoge la preocupación de la autora por la búsqueda de una metodología adecuada y amigable para los estudiantes del curso de Diseño Urbano I en su calidad como docente de la asignatura.

**Arquitecta por la Universidad Ricardo Palma, con estudios de maestría de Transporte Urbano y Docencia Superior, ambas en la Universidad Ricardo Palma; con experiencia en habilitaciones urbanas, construcción de edificaciones residenciales, consultoría en el área de planificación; docente del área de urbanismo de la Universidad Ricardo Palma.
} 


\section{Introducción}

El constructivismo, como enfoque educativo, sirve de marco en esta experiencia para establecer las pautas que orientan el proceso de aprendizaje de los estudiantes de la asignatura Diseño Urbano I. Así, al finalizar la asignatura, el estudiante es competente en aplicar los conocimientos adquiridos previamente sobre la ciudad, en el diseño de nuevos espacios urbanos, renovación y remodelación de los existentes en una estructura urbana, empleando un método disciplinar apropiado.

Las estrategias para lograr competencias en el proceso de aprendizaje son variadas, pero aún no es muy difundida alguna específica para los estudiantes de arquitectura, menos todavía para aquellos que requieren una construcción conjunta motivacional y que a la vez considere su contexto social.

La estrategia de esta intervención se inicia en el conocimiento que ya tiene el estudiante de su contexto social -su barrio-; su lugar cotidiano, espacio de múltiples vivencias; de asociación con múltiples percepciones, de amigos, de compras, de escuela, de juegos, de deportes o simplemente la ubicación de un paradero, un puente que atravesar, etcétera.

El estudiante tiene la oportunidad de trasmitir este conocimiento previo al docente-guía en el transcurso del proceso de la secuencia didáctica de aprendizaje.

Se cumple así la expresión popular que dice: "el que enseña aprende", porque el estudiante se empodera de la construcción del nuevo conocimiento, logra alcanzar habilidades cognoscitivas, desarrolla un comportamiento socio-afectivo, adquiere destrezas motoras y procede al tratamiento de la información. Lo que provoca una construcción interna sólida de la asignatura que involucra aplicaciones en el campo espacial de intervención con efectos que redundan en la seguridad y autoestima del estudiante.

Para medir el incremento pausado, pero acertado en lograr las competencias, se empleó el Modelo de Alineamiento Constructivo desarrollado en la publicación de Biggs, John \&
Tang, Catherine (2007) la taxonomía "SOLO" (Structure of Observed Learning Outcomes) y la estructura de los resultados del aprendizaje observados.

Los resultados permiten inferir que los estudiantes participantes logran mejores niveles de conocimiento, confianza y seguridad para intervenir en las exposiciones finales del semestre; aspecto importante que se asocia con la solidez y profundidad del conocimiento adquirido que le permite establecer un compromiso responsable en su actual contexto social y más adelante con la sociedad en general. Así como una mejor participación en los trabajos de equipo profesional.

\section{El enfoque educativo del constructivismo}

El constructivismo ha ocupado un lugar central en las reformas educativas en las últimas décadas, en muchos países y particularmente en España (Coll, 1987; Carretero, 1993; Pozo, 1996). Se trata de un enfoque educativo que involucra un término con un campo semántico muy complejo, ya que constructivismo se puede entender de diversas maneras, y por ello sería más adecuado hablar de constructivismos.

El constructivismo está asociado a los aprendizajes y conocimientos en los que el individuo reorganiza y reelabora la información recibida con presencia de la conciencia en sus niveles superiores de reflexión, transformándola en una reorganización que tiene una intención clara de lo que se desea alcanzar. Pero también se asocia a otros enfoques, construir con lo que se tiene, con lo que conocemos hoy, con los avances del mañana; lo que implica mucho trabajo por investigar para mejorar, para avanzar en el sentido más profundo que puede contribuir a la realización del Ser humano.

David Ausubel (1963), manifiesta que lo fundamental es conocer las ideas previas de los alumnos y lo que se sabe es que muchas de ellas se obtienen de la percepción de su contexto social. En este caso el barrio, que de- 
viene en el entorno social más próximo del estudiante.

Mientras que los conceptos clave de la teoría de Jean Piaget (1978) son asimilación, acomodación, adaptación y equilibración. Plantea que el mecanismo básico de adquisición de conocimientos consiste en un proceso en el que las nuevas informaciones se incorporan a los esquemas o estructuras preexistentes en la mente de las personas, y que se modifican y reorganizan según un mecanismo de asimilación mental, que consiste en la incorporación de los objetos dentro de los esquemas, que no son otra cosa sino el armazón de acciones que el hombre puede reproducir activamente en la realidad. Y por acomodación se refiere al proceso de modificar esquemas para acomodarse a la nueva información, facilitado por la actividad del ser humano (estudiante).

En este proceso de toma de conocimiento, la equilibración se entiende como el equilibrio entre asimilación y acomodación, que es la adaptación. A través de experiencias acomodadas se promueven nuevos esquemas de asimilación, alcanzándose un nuevo estado de equilibrio. La mente, que es una estructura (cognitiva), tiende a funcionar en equilibrio, aumentando, permanentemente, su grado de organización interna y de adaptación al medio. Estas ideas han servido de punto de partida para las concepciones constructivistas, quienes enfocan al aprendizaje como "un proceso de construcción interno, activo e individual" que cita Carlos Tünnerman (2011) acerca de Piaget.

Por su parte, Lev Vygotski (1979a) introduce el concepto de Zona de desarrollo próximo (ZDP) que se instala entre la Zona de desarrollo real (capacidad de un problema) y la Zona de desarrollo potencial (lo que el sujeto puede resolver con la ayuda de otro). Entre ambas zonas el docente debe intervenir para generar desarrollo.

Ausubel (1983), en su enfoque teórico-abstracto, se apoya en el desarrollo cognitivo y plantea un aporte a la educación cuando sostiene que la base del aprendizaje significativo se sostiene en la vinculación del conocimien- to previo y del nuevo conocimiento, la cuál debe ser simbólica y no arbitraria (no al pie de la letra). Se debe producir un proceso de construcción que permita asimilar el nuevo conocimiento, es decir, llegar a la comprensión más allá del entendimiento (la interiorización antes mencionada). Este proceso de construcción es valioso para uno mismo porque es personal; y depende de la situación, de los intereses, de la motivación y de las experiencias de cada individuo, por ello, dos personas no pueden adquirir un aprendizaje significativo de la misma manera. La percepción juega un papel fundamental en este proceso, siendo muy distinta en cada persona. Además, requiere del lenguaje para lograr el aprendizaje verbal significativo, en este caso, el vocabulario propio de la asignatura.

El individuo que construye el conocimiento es, para cualquier tipo de constructivismo, un sujeto activo que interactúa con el entorno y aunque no se encuentra completamente obligado por las características del medio o por sus determinantes biológicos, va modificando sus conocimientos de acuerdo al conjunto de restricciones internas y externas que tienen una diversidad epistémica: el sujeto individual, el sujeto epistémico, el sujeto psicológico y el sujeto colectivo.

El aprendizaje significativo debe ser gratificante, debe levantar la autoestima del alumno ya que lo incluye activamente en el proceso, explota su potencial y le otorga una autonomía en la cual aprende a aprehender. En esto, también influye el hecho de que el aprendizaje significativo le sirve para ser aplicado en otras situaciones o problemas; lo que significa que es funcional y transferible.

En esta experiencia educativa se considera que la construcción del componente sociocontextual orienta con mayor facilidad los procesos cognitivos del individuo en la búsqueda de logros. J. Anderson (1983) hace hincapié en el constructivismo exógeno, cuando menciona que el aprendizaje se da como consecuencia de conocimientos previos que coadyuvan en la forma de procesar la información y se logra a través de la aplicación efectiva de estrategias corrigiendo conceptos 
erróneos. Con esta postura considera el conocimiento como la reconstrucción de las estructuras que verdaderamente se encuentran en el mundo externo.

Por otro lado, Urie Bronfrenbrenner (1987) en su teoría ecológica del desarrollo humano define un sistema ambiental basado en el desarrollo de los individuos a través de los diferentes ambientes en los que se mueven, los cuales influyen consecuentemente en sus cambios y desarrollo cognitivo, moral y relacional. Entiende al entorno como una serie de estratos interrelacionados, algunos con influencias más cercanas o directas sobre la persona, otros con influencias más lejanas o indirectas; considera además que el entorno es esencialmente dinámico y cambiante.

El aprendizaje según Robert Feldman (2005) es definido como aquel proceso de cambio en el comportamiento de los individuos causada por las experiencias:

En la Educación universitaria donde los estudiantes se van transformando poco a poco con el logro de las competencias específicas propias de su disciplina para el desempeño laboral o empleabilidad como lo denominan en otras latitudes, es importante integrar procesos que se acomoden a las necesidades que se desean lograr en el individuo para participar en la sociedad que los demanda.

En este sentido la enseñanza universitaria incluye un proceso de planificación, liderado por un enfoque que toma en cuenta los diversos aspectos que amalgaman lo endógeno y lo exógeno, entre los cuales se pueden considerar los conocimientos que adquirieron en la asignatura previa, los contenidos y secuencia de la nueva asignatura, los métodos y estrategias de enseñanza, el entorno social, entre otros.

1. Los contenidos de la asignatura: Presentación de diferentes métodos de trabajo para intervenir en la ciudad lo que implica una visión sistémica e integrada, teniendo en cuenta las diferentes etapas de desarrollo de la asignatura. Un inicio con lo conceptual aprendido en el curso an- terior, una instrumentación guiada, una aplicación que requiere pensamiento crítico y reflexión con interacción constante. Una siguiente etapa que presenta mayor complejidad, tanto en lo conceptual como en lo procedimental, al introducir nuevas tecnologías en el desarrollo de su trabajo. La tercera etapa pretende lograr una integración y secuencia temática que se materializa en una aplicación final. La planificación de las sesiones de clases tiene cierta flexibilidad en la medida que se puede reformular en tiempos que permitan una mejor participación más activa y comprometida para el logro de las competencias planteadas para la asignatura. El control del aprendizaje implica que el estudiante haya internalizado con emoción y agrado lo que ha podido desarrollar, y tome conciencia de la utilidad de lo aprendido para su vida profesional.

2. Los métodos y estrategias de enseñanza: La idea clave que debe presidir su elección y articulación es la de ofrecer a los alumnos la oportunidad de adquirir el conocimiento y de practicarlo en un contexto de uso lo más realista posible.

3. En la secuencia de los contenidos se adopta al constructivismo en el diseño instruccional:

- Los alumnos construyen su propia comprensión.

- El nuevo aprendizaje depende de la comprensión actual.

- El aprendizaje se facilita por la interacción social.

- El aprendizaje significativo tiene lugar dentro de tareas de aprendizaje auténticas.

De acuerdo con los principios que se derivan del aprendizaje significativo, se comienza por los elementos más generales y simples para ir introduciendo, progresivamente, los más detallados y complejos.

Además, explica que con la organización social -es decir explotando adecuadamente los 
efectos positivos que pueden tener las relaciones entre los alumnos sobre la construcción del conocimiento, especialmente las relaciones de cooperación y de colaboración- se puede lograr avances significativos tales como que los alumnos se trasladen de su escritorio de trabajo hacia el de otros compañeros para comentar entre ellos los resultados que obtienen y de las diferencias encontradas en el proceso de su trabajo.

En esta experiencia, lo relacionado con la organización social -aspecto tan importante en el enfoque- provoca algunas posiciones controversiales en la medida que los trabajos de cooperación, o de grupo como se les reconoce, requiere que los estudiantes estén en el mismo nivel de conocimientos, sin desfase, y así cumplir con el rol que les corresponde en los trabajos que realizarán, en caso contrario se generan acciones y posiciones "creativas de emergencia" estructuradas por los estudiantes para "solucionar" la actividad correspondiente en el desarrollo del trabajo que realizarán.

\section{Competencias}

En tanto que las competencias describen comportamientos integrados por habilidades cognoscitivas, disposiciones socio-afectivas, destrezas motoras e informaciones que permiten llevar a cabo adecuadamente una actividad (UNESCO, 1999).

Los docentes debemos desarrollar las competencias correspondientes para construirlas conjuntamente con los estudiantes, pudiendo agruparse en las siguientes categorías: Conocer: conjunto estructurado de conocimientos sobre el mundo natural y sociocultural. Implican las disciplinas del currículo que dan lugar a las competencias cognitivas. Hacer: Es un conjunto de procedimientos y estrategias, las competencias procedimentales. Querer: Está referido a motivación, compromiso, esfuerzo; que generan competencias afectivas. Convivir: Es la capacidad para trabajar y relacionarse con compañeros y alumnos, como competencias comunicativas. Y el Ser: Desarrollo del profesor como persona. Responsabilidad ética ante los alumnos, familias y sociedad, como competencias personales (Delors, 1996; Tuning, 2004; García, 2010).

Las competencias suponen precisamente un saber de otra índole, más allá del saber tradicional de la modernidad, un saber que integra el saber con el hacer. En América Latina, el Proyecto Tuning (2004-2007) desarrolla una metodología que pretende determinar puntos de referencia para las competencias genéricas que los estudiantes han de adquirir cuando son estudiantes universitarios, y las específicas de cada disciplina o campo temático. Los puntos comunes de referencia se basan en competencias que respetan la libertad, la diversidad y la autonomía que se identifican en cuatro líneas de trabajo para la metodología Tuning:

- Las competencias (genéricas y específicas) de las áreas temáticas.

- Los métodos de enseñanza, aprendizaje y evaluación.

- Créditos académicos, el cálculo del trabajo de los alumnos, asignación de créditos/asignaturas, etcétera.

- La calidad como parte de un sistema articulado de lo antes mencionado.

El interés por desarrollar competencias en el estudiante implica su capacidad de aprender con mayor exigencia de protagonismo y cuotas más altas de compromiso

De lo anteriormente expuesto se infiere que los procesos de aprendizaje requieren una participación plena del estudiante, lo que es difícil de conseguir en estudiantes con deficiente o bajo nivel de logro en el proceso de aprendizaje. En este caso se trata de presentar una estrategia estructurada para pensar en el estudiante como ser individual primero, y después como integrante de un grupo o de un equipo de trabajo.

\section{Selección de las estrategias didácticas y de aprendizaje}

La estrategia instruccional, según Smith \& Ragan (1999), requiere de tres aspectos: Primero, la forma de organizar secuencialmente el contenido a presentar. Segundo, los medios 
y la agrupación de los estudiantes, y tercero, la manera de obtener los recursos para que se den en la práctica los dos anteriores de acuerdo a lo planificado.

Otros autores como Mayer (1984), Shuell (1988), West, Farmer, Wolff (1991); citados en Díaz-Barriga y Hernández (1999), señalan que las estrategias de enseñanza son procedimientos que promueve el docente para lograr el aprendizaje significativo con éxito de manera autónoma.

El docente en su encuentro presencial y en la forma de modelar la actividad parte de una presentación inicial, guiadas hacia una realización autónoma donde solo se le brinde ayuda al estudiante, estimulando constantemente su aplicación independiente del conocimiento. Para la modificación de la zona de desarrollo próximo de cada uno de los estudiantes estos contenidos deben siempre estar por encima de las posibilidades de ellos. Es decir, el nuevo contenido propuesto será el eslabón siguiente de desarrollo, por tanto, el profesor, para ver la veracidad de las potencialidades de los alumnos, debe controlar los procedimientos que ellos emplean como la ejemplificación, la argumentación, la valoración, las semejanzas, las diferencias, las hipótesis; que posteriormente estarán integrados en un trabajo de grupo. Esto favorece la concepción de tareas del docente, quien apunta a lograr un nivel creciente de profundización en los contenidos.

\section{La estrategia de contexto social}

La idea de la estrategia es sintetizar las diferentes posturas epistemológicas centradas en el aprendizaje del alumno para llevarlas a la acción en el campo de los proyectos de aplicación. El conocimiento del contexto social implica una internalización del área de estudio en el individuo, lo cual le permite realizar un trabajo que desde su inicio puede vislumbrar con mayor acierto; generando confianza y motivación intrínseca en el estudiante. Este es un inicio positivo para materializar las tareas que se desprenden del trabajo emprendido.
Una de las oportunidades que brinda la asignatura de Diseño Urbano I, del área académica de Urbanismo de la carrera de Arquitectura, es la que considera como área de estudio en el entorno urbano; específicamente en el entorno urbano del alumno, esa parte de la ciudad que conoce y reconoce. Este lugar deviene en punto de referencia del aprendizaje y se organiza aún más de acuerdo a las relaciones que establece el alumno en este contexto social. Se centra especialmente en el espacio urbano en el que reside actualmente y que orienta su trabajo semestral; desarrollando las diferentes Unidades de aprendizaje; cada una con la secuencia de contenidos, logros y evaluaciones correspondientes.

Un aspecto relevante que hay que considerar es la participación inicial voluntaria en las exposiciones del primer y segundo trabajo, siendo obligatoria la exposición para finalizar el ciclo para su promoción en la asignatura.

\section{Selección de contenidos y recursos para el aprendizaje}

El rol del estudiante está asociado a la comprensión de su entorno o contexto social; lo que permite mostrar su barrio y como dice el refrán "Cuando enseñas aprendes", válido para alumno y docente en esta estrategia. El estudiante enseña continuamente las particularidades de su barrio, el mismo que "configura" de acuerdo con sus vivencias según lo especificado en las sesiones de cada unidad temática. Todas las modificaciones son posibles ante la presencia de errores.

El estudiante debe controlar los procedimientos que emplea como la ejemplificación, la argumentación, la valoración, las semejanzas, las diferencias y las hipótesis; para una posterior participación en trabajos de grupo ya que favorece la concepción de tareas propuestas por el docente y permiten alcanzar la profundización de los contenidos.

El rol del docente como constructor conjunto del proceso y motivador; continuamente reconoce el área de estudio denominado "MI BARRIO" que configura cada estudiante para las cuatro unidades temáticas de la asignatura 
donde debe crear situaciones de aprendizaje con dos objetivos:

1. Maximizar las oportunidades para que los estudiantes empleen un enfoque profundo.

2. Minimizar las oportunidades de que los estudiantes utilicen un enfoque superficial.

Para lo cual debe estimular constantemente la aplicación independiente del conocimiento, promoviendo la modificación de la zona de desarrollo próximo de cada uno de los estudiantes, incrementando los contenidos para el nivel de reflexión; los cuales deben estar siempre por encima de las posibilidades de ellos, es decir el nuevo contenido propuesto será el eslabón siguiente de desarrollo que ya alcanzaron y también aprovechar las potencialidades de los estudiantes desde la perspectiva de las competencias en cuanto a las dimensiones cognitiva, valorativa y práxica; que se trabajan de manera integral y holística.

\section{Fases del trabajo}

Las fases del trabajo involucran: Habilidades cognoscitivas, comportamientos socio afectivos, psicológicas y destrezas motoras (Ver Figura 1).

1. Habilidades cognoscitivas en los estudiantes de la asignatura de Diseño Urbano I con aprendizaje significativo, se requiere el cumplimiento de ciertas condiciones:

a. La potencialidad de los contenidos: para poder relacionarlos con los anteriores (ideas previas-inclusores-conocimientos de la asignatura Urbanismo II, requisito.)

b. Una estructura cognoscitiva previa del sujeto que permita ser relacionada con los nuevos conocimientos, los subsumidores son los conceptos que vinculan los saberes previos con los nuevos (en este caso el entorno social de cada estudiante).

c. Una disposición significativa del sujeto, expresada en una actitud activa, de atención y motivación para incorporar con sentido un concepto y su aplicación.
(Participación activa en cada sesión de la asignatura)

Este proceso de asimilación cognoscitiva, permite un aprendizaje de manera transformadora, profunda y se da de tres formas:

1. Aprendizaje subordinado: las nuevas ideas se relacionan con ideas relevantes de mayor nivel de abstracción. Las ideas previas sirven de anclaje para las nuevas ideas.

2. Aprendizaje supra ordenado: las ideas relevantes del sujeto tienen menor nivel de abstracción que las nuevas ideas.

3. Aprendizaje combinatorio: la nueva idea es vista en relación con otras ideas preexistentes, pero aquella no es ni más inclusiva ni más específica que estas. Se considera que esta nueva idea tiene algunos atributos de criterio comunes a las ideas preexistentes.

Llevar a la acción este aprendizaje significativo de la Asignatura de Diseño Urbano I, implica la implementación de acciones ordenadas como: (ver figura 2 y 3 )

a. Observar la realidad: Contexto social del estudiante.

b. Elaborar instrumentos de investigación: Data de su barrio en tabla con columnas de información de lo general a lo particular con las características explicadas en clase.

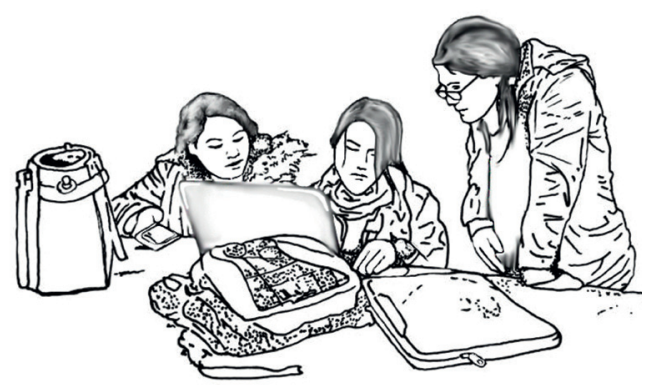

Figura 1. Fases que involucran el trabajo de la Asignatura. Concepto y Diagramación: alumno Diseño Urbano I, Jorge Huamán, julio 2018. 
c. Aplicar instrumentos de investigación: Realizar búsquedas de referencias en los repositorios virtuales de la institución y otros importantes sobre la Unidad temática respectiva.

d. Valorar criterios científicos: Comparar puntos de vista de diferentes expertos o investigadores que han aportado al respecto.

e. Buscar alternativas a la solución de problemas: Promover respuestas alternativas de solución según instrucciones sustantivas del trabajo.

f. Ver los resultados de trabajo: Modalidades de presentación utilizando los TICs en el proceso de avance del trabajo que realiza y que le aportarán al estudiante su fácil y rápida inserción en el campo laboral profesional.
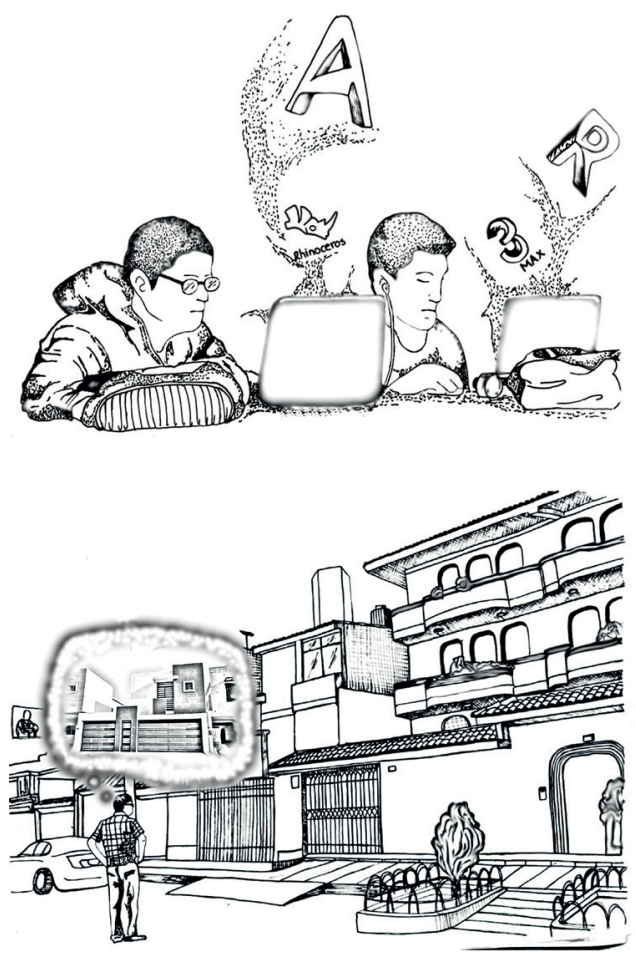

Figura 2 y 3. Implementación de acciones para el aprendizaje significativo de la Asignatura Diseño Urbano I. Alumno Jorge Huamán Vera, julio 2018.
2. Comportamientos socio-afectivo: Desarrollo intrapersonal, desarrollo interpersonal, Comunicación asertiva, capacidad para resolver problemas. A la vez se lleva a cabo un acompañamiento del docente motivador para el incremento de autoestima y de autosuficiencia.

3. Psicológicas: Concentración, control de la ansiedad, confianza y motivación.

4. Destrezas Motoras: Utilización de software para la graficación, exposición y manipulación de imágenes durante el proceso de aprendizaje.

\section{Estrategias de evaluación de resultados de aprendizaje}

Las Competencias de la Asignatura precisan el ser competente, en aplicar los conocimientos adquiridos sobre la ciudad, en el diseño de nuevos espacios urbanos; renovación y remodelación de las preexistencias en una estructura urbana, empleando un método disciplinar apropiado.

Un buen sistema de enseñanza alinea el método y la evaluación de esta con las actividades de aprendizaje establecidas en los logros Biggs \& Tang (2007).

Los resultados de los logros alcanzados, según la estrategia utilizada en el proceso de construcción del conocimiento para esta asignatura, se mide utilizando la taxonomía "SOLO" de John Biggs (2007), del cual se deduce que toda actividad de evaluación es un proceso que consta de tres etapas:

1. Recolección de información, que puede ser o no instrumentada. En este caso es a través de una rúbrica de evaluación por cada Unidad de aprendizaje.

2. Análisis de la información y valoración del resultado de este análisis. En la exposición final de la Asignatura, mostrando la prognosis del área de intervención.

3. Decidir de acuerdo al juicio emitido. Propuestas con argumentación sólida y coherente. 
La asignatura no contempla examen final, solamente examen parcial. Esta estrategia se evalúa en el proceso de aprendizaje, desde el inicio del primer trabajo hasta el último, que desarrolla el estudiante durante el semestre académico.

La evaluación de los aprendizajes, se presenta bajo dos funciones:

1. Una de carácter social de selección y de clasificación, pero también de orientación del alumnado.

2. Una de carácter pedagógico, de regulación del proceso de enseñanza-aprendizaje, es decir, de reconocimiento de los cambios que se han de introducir progresivamente en este proceso para que los alumnos aprendan de forma significativa.
La Taxonomía de Biggs en su "Alineamiento constructivo" considera 5 niveles.

Este modelo de evaluación nos permite alinear el conocimiento profundo para el estudiante de la carrera, el procedimiento o estrategia y la evaluación de los logros alcanzados.

En el caso particular de estudiantes que están en desventaja las evaluaciones de desempeño, se incluye una estrategia motivacional intensa con trabajo personalizado en el aula y con la práctica constante de "saber hacer", lo que implica una fase previa de mostrar lo que ya conoce, buscar información y comparar realidades. Una profunda reflexión respecto de lo que se desea tratar en la Unidad de Aprendizaje correspondiente, que relacionadas con las competencias que se desean alcanzar, el

Tabla 1. Niveles en el alineamiento constructivo de la Taxonomía de Biggs

\begin{tabular}{|c|c|c|c|}
\hline Fase & $\begin{array}{c}\text { Nivel de } \\
\text { comprensión }\end{array}$ & $\begin{array}{l}\text { Acciones } \\
\text { recurrentes }\end{array}$ & Dominio \\
\hline \multirow{3}{*}{ Cuantitativa } & Preestructural & Sin comprensión. & $\begin{array}{l}\text { El estudiante no tiene entendimiento, usa información } \\
\text { irrelevante. }\end{array}$ \\
\hline & Uniestructural & $\begin{array}{l}\text { Identificar, Realizar un } \\
\text { procedimiento sencillo. }\end{array}$ & $\begin{array}{l}\text { Las respuestas solo cumplen con una parte de la ta- } \\
\text { rea, pasa por alto algunos atributos importantes. Estas } \\
\text { respuestas se quedan en la terminología. Identifica un } \\
\text { aspecto relevante, el estudiante tiene destreza en identi- } \\
\text { ficar, seguir un procedimiento y/o recitar. }\end{array}$ \\
\hline & Multiestructural & $\begin{array}{l}\text { Enumerar, Describir, } \\
\text { Hacer una lista, Combi- } \\
\text { nar, Hacer algoritmos. }\end{array}$ & $\begin{array}{l}\text { Puede enfocarse en muchos aspectos relevantes, pero } \\
\text { los considera de forma independiente, no se aborda } \\
\text { la cuestión clave, se elabora de manera más completa } \\
\text { constituiría lo que llaman "contar conocimientos": apa- } \\
\text { bullar con un montón de datos, pero sin estructurarlos. }\end{array}$ \\
\hline \multirow{2}{*}{ Cualitativa } & Relacional & $\begin{array}{l}\text { Comparar, Contrastar, } \\
\text { Explicar causas, Rela- } \\
\text { cionar, Aplicar. }\end{array}$ & $\begin{array}{l}\text { Puede enlazar e integrar muchas partes en un todo co- } \\
\text { herente, los detalles son enlazados a la conclusión y su } \\
\text { significado es entendido, habilidad de relacionar, com- } \\
\text { parar, etcétera. }\end{array}$ \\
\hline & $\begin{array}{l}\text { Abstracto } \\
\text { ampliado }\end{array}$ & $\begin{array}{l}\text { Teorizar, Generalizar, } \\
\text { Formular hipótesis, Re- } \\
\text { flexionar. }\end{array}$ & $\begin{array}{l}\text { La esencia de la respuesta abstracta ampliada es que } \\
\text { trasciende lo dado. El todo coherente se conceptúa en } \\
\text { un nivel superior de abstracción y se aplica a campos } \\
\text { nuevos y más amplios. }\end{array}$ \\
\hline
\end{tabular}

Elaboración basada en John Biggs. Calidad del aprendizaje universitario, Madrid: Narcea, 2005. 
alumno se provee de su entorno cotidiano para facilitar el aprendizaje que abstrae de la complejidad urbana (Ciudad-Barrio).

\section{Algunos resultados de la experiencia realizada en la asignatura}

Asignatura: Diseño Urbano I, asignatura obligatoria del séptimo semestre de la carrera de Arquitectura, que corresponde al Área académica de Urbanismo, de naturaleza teórico práctica, cuatro horas semanales, dos de teoría y dos de práctica.

El requisito para la Asignatura es haber aprobado el curso Urbanismo II, que provee al estudiante del marco teórico de la Ciudad para luego intervenir en el Diseño Urbano creando morfologías sustentadas en un método de planificación para la construcción social.

Población interviniente: matriculados inicialmente 34, retirados a las dos semanas 6 alumnos, permanecen 28 .

Evaluación: Es permanente a través de cinco trabajos prácticos desarrollados durante el semestre académico. El examen parcial es aplicativo, asociado a una investigación extraída de repositorio virtual y a la Unidad Temática correspondiente.

Exposiciones: Corresponden a cada Unidad de aprendizaje y las tres primeras son optativas, pero la participación en ellas incrementa su evaluación final. La exposición final es obligatoria porque permite apreciar la competencia argumentativa que sustenta la propuesta de intervención.

En los dos últimos semestres no se realizaron exposiciones por falta de tiempo y por prórrogas en la entrega de trabajos prácticos.

Resultados de la evaluación: Aprueban 21 estudiantes, promedio máximo 17 , media estadística 14 .

1. Esta asignatura corresponde al estudiante del Plan de Estudios 2006, código de es- tudiante 2014xxxx, pero en los semestres 2016-I, 2016-II, y 2017-I, participaron estudiantes de otros semestres, distintos al semestre de estudios del curso. Lo llevaron estudiantes con códigos del 2007, 2008 y 2011 inclusive; razón por la cual se establece una estrategia adecuada para esta situación particular.

2. Se trabaja constructivamente docentealumno en el aula. El desarrollo de los trabajos se realiza en las horas de práctica de la asignatura, con mayor porcentaje de asistencia en este horario de práctica; situación opuesta para las horas de teoría.

3. Los estudiantes llegan a la asignatura con carencias de un vocabulario idóneo, escasa reflexión crítica, bajo nivel de esquematización, poca claridad en las ideas que orientan su propuesta de intervención, etcétera.

4. Un conocimiento superficial de programas para la manipulación de imágenes, útil para la propuesta final.

5. En las exposiciones iniciales pocos alumnos participan, luego se van incrementando hasta el final.

6. Los estudiantes que llevan a cabo la construcción secuencial del trabajo en horas de clase y cumplen con los requisitos de entrega, y presentan todos los trabajos programados, son promovidos.

7. La asistencia semanal en la realización de los trabajos en clase se constituye en factor decisivo para conseguir las competencias propuestas.

8. El avance reflexivo y constante marcan la pauta de la responsabilidad que asume el estudiante en la construcción de sus habilidades cognoscitivas.

9. Interacción constante en el desempeño de su trabajo durante las orientaciones del docente motivador y constructor conjunto del aprendizaje.

10. Originalidad en la presentación de su trabajo, cumpliendo con los requisitos establecidos. 


\section{Referencias}

Anderson, J. (1983). The architecture of cognition. Cambridge, Ma: Harvard University Press.

Ausubel, D. (1963). The psychology of meaningful verbal learning. New York: Grune and Stratton.

Ausubel, D. \& Hanesian, H. (1983). Psicología Educativa: Un punto de vista cognoscitivo. $2 \mathrm{a}$ ed. México: Trillas.

Biggs, J. \& Tang, C. (2007). Teaching for Quality Learning at University Editor s.1.: Open University Press.

Bronfrenbrenner, U. (1987). La ecología del desarrollo humano. Experimentos en entornos naturales y diseñados. Barcelona: Paidós.

Carretero, M. (1993). Constructivismo y educación. Zaragoza: Edelvises.

Coll, C. (1987). Psicología y Curriculum. Barcelona: Laila.

Delors, J. (1996). Los cuatro pilares de la educación. En UNESCO, La educación encierra un tesoro. Informe a la UNESCO de la Comisión internacional sobre la educación para el siglo XXI. Madrid, España: Santillana.

Díaz-Barriga, F. \& Hernández, G. (1999). Estrategias docentes para un aprendizaje significativo. Una interpretación constructivista. 2a ed. México: Mac Graw-Hill Interamericana.

Feldman, R. (2005). Aprendizaje con poder: Estrategias para el éxito en la universidad y en la vida. México: Mac Graw-Hill Interamericana de México.

García, E. (2010). Competencias éticas del profesor y calidad de la educación. Revista Electrónica Interuniversitaria de Formación del Profesorado, $13(4)$.
Novak, J. (1998). Conocimiento y aprendizaje. Madrid: Alianza Editorial.

Pérez de Celis, M. (2013). Estrategias de enseñanza para el aprendizaje por competencias con enfoque constructivista sociocultural. México: Facultad de Ciencias de la Computación, Benemérita Universidad Autónoma de Puebla.

Piaget, J. (1978). La Equilibración de las estructuras cognitivas. Problema central del desarro1lo. Madrid: Siglo XXI.

Pozo, I. (1996). Psicología y Educación. Madrid: Alianza Editorial.

Serrano, J. y Pons, R. (2011). El constructivismo hoy: enfoques constructivistas en educación. Revista Electrónica de Investigación Educativa.

Smith, P. \& Ragan, T. (1999). Instrutional design. New York: John Wiley \& Sons.

Tünnerman, C. (2011). El Constructivismo y aprendizaje de los estudiantes en UDUAL, México, 48, enero-marzo, pp. 21-32.

Tuning-America Latina 2004-2007. (2007). Reflexiones y perspectivas de la educación superior en América Latina, Informe Final Proyecto. Bilbao, España: Publicaciones de la Universidad de Deusto.

UNESCO (1999). Declaración Mundial sobre la educación superior en el siglo XXI: Visión y acción.

Vygotsky, L. (1979a). El desarrollo de los procesos psicológicos superiores. Barcelona: Crítica.

Vygotsky, L. (1979b). Psicología y Pedagogía. Madrid: Akal. 